Sir,

\section{Irreversible neurotoxicity with acyclovir treatment of acute retinal necrosis}

Neurotoxicity is a rare but recognised side-effect of acyclovir, which typically occurs in those with cancer or renal failure. We report a case of hand paraesthesia in a man who received intravenous acyclovir for acute retinal necrosis. This case is unusual in that it occurred in a healthy man and its effects did not completely reverse.

\section{Case report}

A previously healthy right-handed 50-year-old toolmaker presented to us with a 10 day history of blurred vision in his right eye. Visual acuity in this eye was $6 / 18$ unaided, improving to $6 / 9$ with a pinhole, and there was a granulomatous anterior uveitis, papillitis, retinal necrosis, localised retinal detachment and vasculitis. The left eye was never involved. He had no history of neurological disease, previous hand trauma and was on no medication.

Acute retinal necrosis was diagnosed and the patient was admitted and received a week's course of intravenous acyclovir $850 \mathrm{mg}$ t.d.s. ( $32 \mathrm{mg} / \mathrm{kg} /$ day) plus $80 \mathrm{mg}$ prednisolone orally. The intravenous cannula was placed in a vein in the antecubital fossa of his left hand. Approximately 3 days later he developed numbness and tingling over the palmar surface and fingers of his right hand. The patient did not inform the staff of these symptoms and the acyclovir was continued. His ocular condition improved and he was discharged on valcyclovir $1 \mathrm{~g}$ t.d.s. (which was continued for 6 weeks) and prednisolone $60 \mathrm{mg}$ for the right eye.

Having stopped the acyclovir his neurological symptoms improved over 3-4 months but currently, 2 years later, he still suffers residual stable paraesthesia. Current neurological assessment indicates reduced pinprick sensation over the ulnar region of the right palmar surface but no muscle weakness. Unaided visual acuity in his right eye is counting fingers improving to $3 / 60$ with a pinhole.

\section{Comment}

Acyclovir selectively inhibits viral DNA polmerase. Its side-effects include neurotoxicity, ${ }^{1-7}$ nephrotoxocity ${ }^{8}$ and hypotension. ${ }^{9}$ Neurological effects include alterations in mental status (commonest), myoclonic jerks, action tremors, hallucinations, convulsions, ataxia, dysarthria and hemiparaesthesia. ${ }^{1}$ In Haefeli et al.'s ${ }^{4}$ review of 24 patients only $13 \%$ of the reported side-effects were unilateral focal symptoms. Unlike this case, reversibility of side-effects is characteristic and occurred in all the case reports reviewed by Adair et al. ${ }^{1}$
Our patient was healthy but in virtually all reported cases of neurotoxicity the patients either had renal disease or cancer. ${ }^{1-3,5}$ Those 'healthy' patients who develop neurotoxicity may well have associated impaired renal function secondary to the acyclovir. ${ }^{8}$ This would suggest that baseline and regular tests or renal function are merited in those given intravenous and possibly oral acyclovir. Kitching et al. ${ }^{2}$ suggest that for patients with renal disease receiving acyclovir, lower dosages should be given than those recommended. The total dose of intravenous acyclovir that our patient received was $17.85 \mathrm{~g}$. The recommended threshold concentration for developing neurotoxicity is $4.5 \mu \mathrm{g} / 1$, but 4 of the 17 cases reviewed by Adair et al. ${ }^{1}$ developed side-effects at a lower concentration, and this suggests that it is the rate of renal excretion as opposed to the absolute dose which is more significant. In addition, the serum concentration of acyclovir can be misleading, as there is a delay before equilibrium is reached between drug concentrations in serum and cerebrospinal fluid. ${ }^{6}$ Adair et al.'s review ${ }^{1}$ indicates that the majority of cases of neurotoxicity were secondary to those receiving intravenous as opposed to oral acyclovir. It is unclear how acyclovir causes neurotoxicity, but there is indirect evidence to suggest a demyelinating process. ${ }^{5}$

The differential diagnosis when acyclovir neurotoxicity is suspected includes herpes encephalitis. Helpful features suggesting neurotoxicity include absent signs of meningeal irritation, normal cerebrospinal fluid, normal CT findings and an abnormal electroencephalogram. ${ }^{1-3}$ If the diagnosis is still in doubt a trial of haemodialysis to see whether the condition improves is helpful. ${ }^{3}$

This case illustrates the potential neurological side-effects of acyclovir, which may be irreversible. They can occur using oral acyclovir in those with no general medical problems, but are commoner with the intravenous format, particularly if there is associated nephropathy.

\section{References}

1. Adair JC, Gold M, Bond RE. Acyclovir neurotoxicity: clinical experience and review of the literature. South Med J 1994;87:227-31.

2. Kitching AR, Fagg D, Hay NM, Hatfield PJ, MacDonald A. Neurotoxicity associated with acyclovir in end stage renal failure. N Z Med J 1997;110:167-9.

3. Davenport A, Goel S, Mackenzie JC. Neurotoxicity of acyclovir in patients with end-stage renal failure treated with continuous ambulatory peritoneal dialysis. Am J Kidney Dis 1992;20:647-9.

4. Haefeli WE, Schoenenberger RAZ, Weiss P, Ritz RF. Acyclovir-induced neurotoxicity: concentration-side effect relationship in acyclovir overdose. Am J Med 1993;94:212-5.

5. Wade JC, Meyers JD. Neurologic symptoms associated with parenteral acyclovir treatment after marrow transplantation. Ann Intern Med 1983;98:921-5. 
6. Bean B, Aeppli D. Adverse effects of high dose intravenous acyclovir in ambulatory patients with acute herpes zoster. J Infect Dis 1985;151:362-4.

7. Feldman S, Rodman J, Gregory B. Excessive serum concentrations of acyclovir and neurotoxicity. J Infect Dis 1988;157:385-8.

8. Becker BN, Schulman G. Nephrotoxicity of antiviral therapies. Curr Opin Nephrol Hypertens 1996;5:375-9.

9. Dorsky DI, Crumpacker CS. Drugs five years later: acyclovir. Ann Intern Med 1987;107:859-74.

Daragh K. Fahey

Quresh Mohammed

Chris Canning

Eye Unit

Southampton General Hospital

Tremona Road

Southampton SO16 6YD, UK

Sir,

Ptois as presenting sign of metastatic skin melanoma Metastases of cutaneous melanoma to the orbit have been reported several times, ${ }^{1}$ frequently in combination with widespread systemic malignancy. ${ }^{2}$ The clinical signs and symptoms may vary, including diplopia or enophthalmos. ${ }^{3}$ This report describes a patient with a history of cutaneous melanoma who developed unilateral ptosis as the first sign of metastatic skin melanoma.

Case report

A 64-year-old woman was referred with ptosis of the left upper eyelid of 2 months' duration (Fig. 1a). She did not complain about diplopia or loss of vision. One month previously, a nodular melanoma Clark level III, Breslow thickness $2.0 \mathrm{~mm}$, had been removed from her left forearm.

On examination the vertical eyelid fissure measured from the lower limbal margin was $9 \mathrm{~mm}$ on the right and $3 \mathrm{~mm}$ on the left. Levator muscle function was $12 \mathrm{~mm}$ on the right and $5 \mathrm{~mm}$ on the left side. Exophthalmometry was $16 \mathrm{~mm}$ on the right and $17.5 \mathrm{~mm}$ on the left. Eye movements showed slight limitations in abduction, adduction and elevation of the left eye. A solid mass could be palpated between the eyeball and superior orbital margin on downgaze.

Funduscopy revealed indentation of both upper quadrants of the left eye. A CT scan showed a tumour in the left levator muscle with a downward shift of the globe (Fig. 1b). Moreover, there were cerebral lesions, suggestive of metastatic disease.

At surgical exploration, a brown-black mass was found infiltrating and replacing the levator muscle. An excisional biopsy specimen measuring $3 \times 1.5 \times 1.5 \mathrm{~cm}$ was removed and examined (Fig. 2a). Histological examination showed a malignant melanoma morphologically identical to the cutaneous melanoma excised earlier (Fig. 2b). Additional tumour clonality analysis was performed by identifying $X$-chromosome inactivation with a polymorphic DNA marker. DNA was extracted from microdissected tissue fragments of the cutaneous and the orbital tumour. Identical loss of two

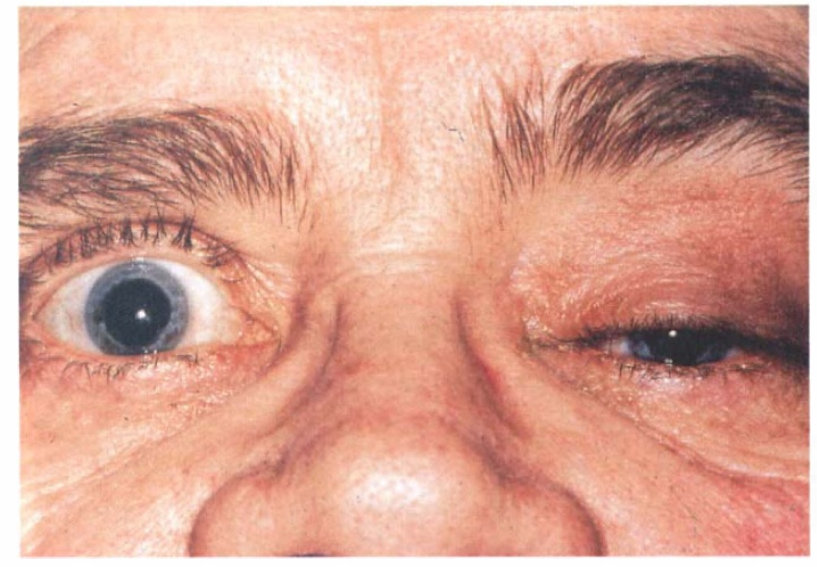

(a)

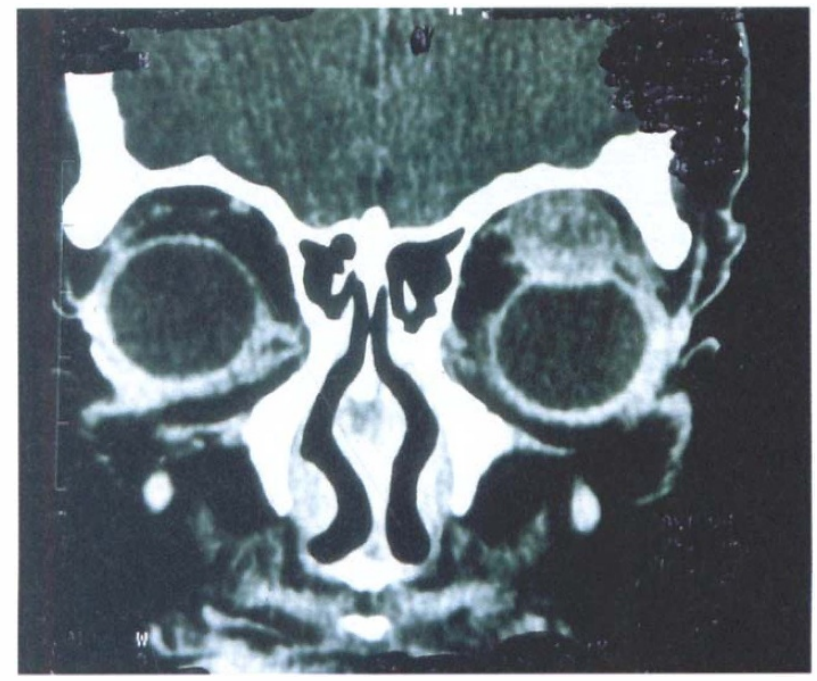

(b)

Fig. 1. (a) Patient at presentation. (b) Axial CT scan showing a mass in the left levator muscle with indentation and downward shift of the globe.

polymorphic markers was found, which is consistent with origin from a single tumour. Further systemic examination revealed metastases in the axillary lymph nodes, the skin of the back and the brain. Our patient died 6 months after orbital surgery due to cerebral metastases.

\section{Comment}

Cutaneous melanomas larger than $1.5 \mathrm{~mm}$ are notorious for fast distant spread and metastatic death. Metastasis occurs by lymphatic and haematogenous spread. Predilection sites are the lung, central nervous system and liver, but rarely the orbit. ${ }^{4}$

In our case, a metastasis of this malignancy infiltrated the left levator muscle which led to ptosis of the upper lid. Ptosis secondary to orbital metastases of skin melanoma has only been reported in two patients by Orcutt and Char, ${ }^{2}$ but this is - in our opinion - the first report of ptosis as the presenting sign.

Recent-onset unilateral ptosis in a patient with a history of neoplastic disease, e.g. cutaneous melanoma, should alert the clinician to the possibility of orbital metastasis. 